\title{
Tetramethylpyrazine reduces blood-brain barrier permeability associated with enhancement of peripheral cholinergic anti-inflammatory effects for treating traumatic brain injury
}

\author{
AIMIN WANG ${ }^{*}$, GUANGBIN ZHU* ${ }^{*}$ PING QIAN and TAO ZHU \\ Department of Intensive Care Unit, Taicang Affiliated Hospital of Soochow University, Taicang, Jiangsu 215006, P.R. China
}

Received June 23, 2016; Accepted April 28, 2017

DOI: $10.3892 /$ etm.2017.4754

\begin{abstract}
Traumatic brain injury (TBI) is a diverse group of intracranial injuries resulting from external mechanical insults to the brain. While basic and clinical research for TBI has been conducted for decades, it has not identified cost-effective medical interventions for treating TBI. Tetramethylpyrazine (TMP), which is derived from the Chinese herb, Ligusticum chuanxiong Hort (Chuan Xiong), has been clinically used for treating ischemic brain injury for years. However, whether TMP could provide effective benefits for improving the outcomes following TBI is unknown. In the present study, using controlled cortical impact (CCI) injury to create an animal model of TBI, the potential effects of TMP on improving blood-brain barrier (BBB) permeability in the early phase of the secondary injury, as well as the splenic anti-inflammatory activities, were evaluated. Cognitive functions were also assessed by Morris water maze trials following TBI. Results demonstrated that, at $24 \mathrm{~h}$ after CCI injury, BBB permeability was significantly reduced $(\mathrm{P}<0.05)$ by the application of TMP. In addition, within $24 \mathrm{~h}$ after CCI injury, the plasma levels of interleukin (IL)-1 $\beta$ and tumor necrosis factor (TNF)- $\alpha$, and protein and mRNA expression levels of IL-1 $\beta$ and TNF- $\alpha$ in the spleen were significantly lowered by TMP $(\mathrm{P}<0.05)$. Furthermore, within $24 \mathrm{~h}$ after CCI injury, the activation of the splenic anti-inflammatory effects mediated by nicotinic acetylcholine receptor $\alpha 7$ (nAChRa7) stimulation were significantly enhanced by TMP $(\mathrm{P}<0.05)$. Additionally, impaired spatial memory acquisition and consolidation were significantly improved by TMP after CCI injury $(\mathrm{P}<0.05)$. Together, in light of these data, in the treatment of TBI, TMP could effectively reduce BBB permeability,
\end{abstract}

Correspondence to: Professor Tao Zhu, Department of Intensive Care Unit, Taicang Affiliated Hospital of Soochow University, 58 South Changsheng Road, Taicang, Jiangsu 215006, P.R. China E-mail: zhutao0512@hotmail.com

*Contributed equally

Key words: tetramethylpyrazine, traumatic brain injury, blood-brain barrier, anti-inflammation which may be closely associated with the enhanced splenic anti-inflammatory effects activated by nAChRa7 stimulation, and potentially improve cognitive recovery concerning spatial learning and memory.

\section{Introduction}

Traumatic brain injury (TBI) is a diverse group of intracranial injuries resulting from the transduction of external energy into the brain, which exceeds its own protective capacity (1). It was estimated that $\sim 10$ million people sustain TBI worldwide annually, leading to the burden of mortality and morbidity imposing on society (2). In 2009, within the USA alone, more than 2.4 million people were affected by TBI, which contributed to one-third of all injury-related mortality, and $~ 5.3$ million people lived with disability from TBI (3). Individuals who survive severe TBI require 5-10 years of intensive therapy, and the aggregate annual costs in 2010 for TBI were estimated to be $\sim \$ 76.5$ billion in the USA $(3,4)$. Although there has been extensive basic and clinical research for TBI, it has failed to identify effective approaches validated by large randomized control trials to reduce the pathology and improve the clinical outcomes following TBI, particularly for severe cases (5). Except for the surgical interventions and supportive therapies, such as brain swelling control, only treatment with the $N$-methyl-D-aspartate receptor antagonist, amantadine, in the sub-acute phase has been evidenced to promote the functional recovery of TBI patients (6). Accordingly, to further investigate and develop cost-effective medical interventions for treating TBI would be of critically importance.

Ligusticum chuanxiong Hort, also known as L. wallichii Franchat (Chuan Xiong), belongs to the family of Umbelliferae and its rhizome part has been traditionally applied for treating a range of nervous and cardiovascular system diseases in Traditional Chinese Medicine for hundreds of years (7). To date, $>200$ compounds have been isolated from this plant, which may be categorized into five major groups, namely phenols and organic acids, phthalides, alkaloids, polysaccharides, ceramides and cerebrosides (8). Among them, 2,3,5,6-tetramethylpyrazine (TMP), which belongs to the alkaloids group (also known as Ligustrazine), is considered to be one of the most important bioactive ingredients from the herb Chuan Xiong, and has been clinically used in the treatment of ischemic brain injury for years (9). In fact, it has been 
widely studied that TMP may exert explicit neuroprotective roles, such as anti-inflammatory, antioxidant and anti-apoptotic effects, for treating ischemic stroke (10-13). However, the beneficial effects of TMP on improving the outcomes of TBI have not been fully elucidated.

It is generally understood that the blood-brain barrier (BBB) serves as the key structure for anatomically isolating the delicate structure of brain parenchyma from its surrounding blood and fluid spaces, and its integrity is essentially important for maintaining the homeostasis of the nervous system (14). In the early phase after TBI, the complex secondary injury cascades induced by the initial external mechanical insults may lead to the breakdown of BBB mediated by various cellular and molecular events, such as the dysfunction of the neuro-glio-vascular unit, endothelial disruption and derangement of tight junction proteins (15). Consequently, the transiently increased $\mathrm{BBB}$ permeability would result in the uncontrolled leakage of fluids and inflammatory cells extravasated from the blood into the affected brain tissue, so as to further deteriorate other secondary TBI pathologies, including decreased cerebral blood flow, excitotoxicity, neuroinflammation and synaptic reorganization $(15,16)$. Previous research has indicated that TMP could effectively reduce BBB permeability in ischemic brain injury (17). However, whether and to what extent the breakdown of BBB following TBI could be potentially improved by TMP is largely unclear.

Increasing research has revealed that BBB permeability in TBI could be reciprocally regulated in response to the inflammatory status of either central or peripheral origin $(15,18)$. Following TBI, the phenotype-shifted neurons and surrounding glia produce a range of pro-inflammatory cytokines, such as interleukin (IL)-6, tumor necrosis factor- $\alpha$ (TNF- $\alpha$ ) and IL-1 $\beta$ (19). Simultaneously, the stimulation of the hypothalamic-pituitary-adrenal axis may further adrenergically activate the splenic macrophages to secrete pro-inflammatory cytokines into the systemic circulation (20). Eventually, these pro-inflammatory cytokines may directly lead to the recruitment and degranulation of neutrophils, so as to degrade the extracellular matrix by proteases and increase BBB permeability (15). Notably, studies have demonstrated that the vagus nerve activity, particularly the splenic component, may exert the cholinergic anti-inflammatory effects on improving the breakdown of BBB after TBI, mediated by the stimulation of the nicotinic acetylcholine receptor $\alpha 7$ (nAChRa7) subunit on splenic macrophages (21-23). Novel findings have indicated that TMP may elicit certain anti-inflammatory roles for treating ischemic brain injury and neurodegenerative diseases $(24,25)$. Nevertheless, the regulation of inflammation by TMP, particularly the splenic anti-inflammatory effects, have not been investigated.

The present study utilized a rat model of controlled cortical impact (CCI) injury in order to determine whether, and to what extent, increased BBB permeability following TBI could be improved by TMP. Furthermore, whether splenic cholinergic anti-inflammatory effects could be regulated by TMP was also investigated. Results demonstrated that TMP could effectively reduce CCI-induced BBB permeability, exerting neuroprotective roles for treating TBI. These effects may be closely associated with the enhancement of splenic cholinergic anti-inflammatory effects.

\section{Materials and methods}

Experimental animals and drug administration. A total of 48 male Sprague Dawley rats (age, 6 months; weight, 200-250 g) were supplied by the Laboratory Animal Research Center of Soochow University (Taicang, China). Rats were maintained under standard conditions for at least 1 week, and individually housed in plastic cages in an animal room under temperature-controlled conditions of $22-25^{\circ} \mathrm{C}$ and relative humidity of 50-60\% with a 12-h light/dark cycle and access to commercial pellets and water ad libitum. Animals were randomly assigned to three groups: TMP group $(n=18)$ (among which 6 rats were sacrificed at $24 \mathrm{~h}$ after CCI, 6 rats were injected by TMP from day 1 to day 14 after CCI, and 6 rats were injected by TMP from day 2 to day 14 after CCI), vehicle group $(n=18)$ (among which 6 rats were sacrificed at $24 \mathrm{~h}$ after CCI, 6 rats were injected with saline from day 1 to day 14 after $\mathrm{CCI}$, and 6 rats were injected with saline from day 2 to day 14 after CCI) and sham group $(n=12)$ (as described in the next section; among which 6 rats were sacrificed at $24 \mathrm{~h}$ after sham operation and 6 rats were used for Morris water maze experiment). Experiments and drug administration were performed in a double-blind manner. TMP (Sigma-Aldrich; Merck KGaA, Darmstadt Germany) (20 mg/kg) for the TMP group, and an equivalent volume of saline (Sigma-Aldrich; Merck KGaA) for the vehicle group, was injected intraperitoneally twice daily for 14 days. The first injection was administered $30 \mathrm{~min}$ after the impact procedure, as described below. For the assessment of cognitive recovery (i.e. Morris water maze (MWM) trial), deprivation of injection administration on the same day of the TBI procedure was specified as TMP (2-14) group $(n=6)$ and vehicle (2-14) group $(n=6)$. All procedures were approved by the Soochow University Animal Ethics Committee and conformed to the Guide for the Care and Use of Laboratory Animals.

Surgical procedure for CCI injury model. The CCI model for experimental traumatic TBI was performed using an electromagnetic controlled pneumatic impact device (Precision Systems and Instrumentation, Fairfax Station, VA, USA) by driving a piston at a preset speed onto the exposed surface of the brain. The depth of compression was controlled by zeroing the piston on the surface of the rat brain. Under anesthetizing with intraperitoneal injections of $3 \%$ sodium pentobarbital $(50 \mathrm{mg} / \mathrm{kg}$ ) and under sterile conditions, a midline longitudinal incision was performed on the rat skull and then the craniotomy (6-mm in diameter) was made over the left parietal cortex using a portable drill and trephine. The injury consisted of a single impact at the velocity of $6.0 \mathrm{~m} / \mathrm{sec}$ and a depth of $5 \mathrm{~mm}$ delivered to the left parietal cortex. Subsequently, the scalp was covered using cyanoacrylate tissue glue. Following surgery, rats were allowed to recover in a warmed chamber and then returned to their cages. Throughout the procedure, rat body temperature was monitored and maintained at $37.0 \pm 0.5^{\circ} \mathrm{C}$. TBI group rats were subjected to the complete CCI surgical procedure, whereas the vehicle group rats were anesthetized and received the incision but not the craniotomy or impact procedure. For sham group $(n=12)$, skull midline longitudinal incision and craniotomy was performed without TBI, then the scalp was covered using cyanoacrylate tissue glue. 
Measurement of levels of plasma IL-1 $\beta$ and TNF- $\alpha$. Blood samples $(0.5 \mathrm{ml}$ each) were taken from an intravenous tube implanted in the femoral vein at 4,12 and $24 \mathrm{~h}$ after CCI injury, respectively. With the use of EDTA as an anticoagulant, plasma was prepared by centrifugation at $2,000 \mathrm{xg}$ at $4^{\circ} \mathrm{C}$ for $10 \mathrm{~min}$ to remove the erythrocytes, leukocytes and platelets. The plasma was aliquoted and frozen at $-80^{\circ} \mathrm{C}$ until further use. Plasma concentrations of IL- $1 \beta$ and TNF- $\alpha$ were detected using commercially available ELISA kits (IL-1 $\beta$ kit cat. no. D730238-0048, TNF- $\alpha$ kit cat. no. D730457-0048; Sangon Biotech Co., Ltd., Shanghai, China), according to the manufacturer's instructions.

Evaluation of $B B B$ permeability. BBB permeability was determined by measuring the extravasation of Evans Blue dye in each group. In TMP, vehicle, and sham groups, 6 rats were randomly selected to be sacrificed in each group for BBB permeability assay. At $24 \mathrm{~h}$ after CCI injury, rats were anesthetized with intraperitoneal injections of $3 \%$ sodium pentobarbital $(50 \mathrm{mg} / \mathrm{kg})$ and $3 \%$ Evans Blue $(4 \mathrm{ml} / \mathrm{kg})$ was injected slowly into the jugular vein and allowed to circulate for $1.5 \mathrm{~h}$ before sacrifice. To maintain unconsciousness until decapitation, rats were also intraperitoneally injected with $3 \%$ ketamine $(25 \mathrm{mg} / \mathrm{kg}) 1 \mathrm{~h}$ after sodium pentobarbital injection. Then, rats were transcardially perfused with phosphate-buffered saline (PBS) followed by $4 \%$ paraformaldehyde for removing all the intravascular dye until the drainage was colorless. Finally, rats were sacrificed by decapitation. The ipsilateral hemispheres were then placed in $5 \mathrm{ml} 4 \%$ paraformaldehyde and incubated at room temperature for $48 \mathrm{~h}$. The amount of Evans Blue dye in the supernatant was measured by absorbance of the supernatant using a spectrophotometer (Analytik Jena AG, Jena, Germany) at a wavelength of $632 \mathrm{~nm}$. A linear standard curve was created based on Evans Blue external standards. The tissues were then dried at $95^{\circ} \mathrm{C}$ for 5 days. Based on the standard curve, the amount of Evans Blue was quantified as $\mu \mathrm{g}$ of Evans Blue per mg dry weight of brain tissue.

Western blot analysis. At $24 \mathrm{~h}$ after CCI injury, 6 rats in each group were sacrificed and protein samples were routinely extracted from spleen tissues. Tissues were routinely homogenized in protein extraction buffer (NP-40 lysis buffer; Thermo Fisher Scientific, Inc.) with freshly added protease inhibitors using sonication. After centrifugation of samples at $12,000 \mathrm{xg}$ for $20 \mathrm{~min}$ at $4^{\circ} \mathrm{C}$, the supernatant was collected and protein concentration was determined by Bradford method (Quick Start $^{\mathrm{TM}}$ Bradford Protein assay; Bio-Rad Laboratories, Inc., Hercules, CA, USA). Protein samples ( $80 \mu \mathrm{g})$ were then separated by $0.1 \%$ sodium dodecylsulfate and $10 \%$ polyacrylamide separating gel with $4 \%$ polyacrylamide stacking gel and transferred to immobilon-P polyvinyl difluoride membranes (EMD Millipore, Billerica, MA, USA). Each membrane was incubated with Tris-buffered PBS supplemented with 5\% bovine serum albumin (Sigma-Aldrich; Merck KGaA) and $0.1 \%(\mathrm{v} / \mathrm{v})$ Tween-20 (Sigma-Aldrich; Merck KGaA) with gentle shaking for $1 \mathrm{~h}$ at room temperature. Following this, membranes were incubated with primary rabbit polyclonal antibodies against nAChRa7 (ab-10096; 1:1,000; Abcam, Cambridge, MA, USA), nuclear factor (NF)-кB (p65; sc-109; 1:1,000; Santa Cruz Biotechnology, Inc., Dallas, TX, USA),

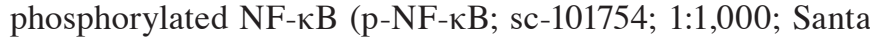
Cruz Biotechnology, Inc.), signal transducer and activator of transcription 3 (STAT3; sc-482, 1:1,000; Santa Cruz Biotechnology, Inc.), p-STAT3 (sc-7993; 1:1,000; Santa Cruz Biotechnology, Inc.), IL-1 $\beta$ (sc-7884; 1:1,000; Santa Cruz Biotechnology, Inc.) and TNF- $\alpha$ (sc-374186; 1:1,000; Santa Cruz Biotechnology, Inc., Dallas, TX, USA), respectively, at $4^{\circ} \mathrm{C}$ overnight. Subsequently, the membranes were washed three times for $10 \mathrm{~min}$ with Tris-buffered saline-Tween-20 solution and further incubated at room temperature with secondary horseradish peroxidase-conjugated goat anti-rabbit antibodies (A0545; 1:5,000; Sigma-Aldrich; Merck KGaA). Proteins were then visualized using enhanced chemiluminescent reagents (GE Healthcare, Chicago, IL, USA) and exposed to an X-ray film. $\beta$-actin protein (sc-47778, 1:1,000; Santa Cruz Biotechnology, Inc.) was blotted on the same membrane as an internal control for normalizing the relative density. Results were quantified and analyzed with a Kodak Electrophoresis Documentation and Analysis System and its ID image analysis software version 2.0 (Kodak, Rochester, NY, USA).

Reverse transcription-quantitative polymerase chain reaction ( $R T-q P C R)$. At $24 \mathrm{~h}$ after CCI injury, and after rats were sacrificed, total RNA was isolated from spleen tissues by homogenizing with TRIzol reagent (Invitrogen; Thermo Fisher Scientific Inc., Waltham, MA, USA), followed by adding chloroform. Then, after the homogenate was separated by centrifugation at $12,000 \mathrm{x}$ g for $10 \mathrm{~min}$ at $4^{\circ} \mathrm{C}$, RNA was precipitated from the upper aqueous layer with isopropanol. from spleen tissues. cDNA synthesis was performed using an MMLV-RT reverse transcriptase kit (cat. no. 28025013; Invitrogen; Thermo Fisher Scientific, Inc.). qPCR analysis was employed to determine nAChRa7, IL-1 $\beta$ and TNF- $\alpha$ gene expression levels in spleen tissues. GAPDH was used as an internal reference and serial dilutions of the positive control were performed on each plate to create a standard curve. The primer sequences used were as follows: Rat $\mathrm{nAChRa7}$, forward 5'-GAGGCTGTACAAGGAGCTGG-3' and reverse 5'-ACC CTCCATAGGACCAGGAC-3'; rat IL-1 $\beta$, forward 5'-GAC TTCACCATGGAACCCGT-3' and reverse 5'-GGAGACTGC CCATTCTCGAC-3'; rat TNF- $\alpha$, forward 5'-GGCTTTCGG AACTCACTGGA-3' and reverse 5'-CCCGTAGGGCGATTA CAGTC-3'; and rat GAPDH, forward 5'-GGTGGTCTCCAC GGACTTTA-3' and reverse 5'-CAAGGAGGGGCCTTTATT TC-3'. qPCR (iQ ${ }^{\mathrm{TM}} \mathrm{SYBR}^{\circledR}$ Green Supermix, cat. no. 170-8882; Bio-Rad Laboratories, Inc.) was performed using a Bio-Rad iCycler iQ $25 \mu 1$ system (Bio-Rad Laboratories, Inc.) in a 96-well plate (Takara Biotechnology Co., Ltd., Dalian, China). The thermocycling conditions were as follows: $42^{\circ} \mathrm{C}$ for $30 \mathrm{~min}, 95^{\circ} \mathrm{C}$ for $15 \mathrm{~min}$, followed by 40 cycles of $95^{\circ} \mathrm{C}$ for $20 \mathrm{sec}, 56^{\circ} \mathrm{C}$ for $1 \mathrm{~min}$ and $72^{\circ} \mathrm{C}$ for $20 \mathrm{sec}$. The amount of target gene was normalized to the reference GAPDH to obtain the relative threshold cycle. The $2^{-\Delta \Delta \mathrm{Cq}}$ method (26) was used to determine the relative abundance of target gene expression in each group.

Measurement of spatial learning and memory. The Morris water maze (MWM) was applied to assess spatial learning and memory following TBI injury. In each group, half of the rats were sacrificed, while the other half rats were maintained to 
be used for the MWM trials. In TMP group, rats $(n=6)$ injected by TMP from day 1 to day 14 and those $(n=6)$ injected by TMP from day 2 to day 14 after CCI were both randomly selected for MWM trial. In vehicle group, rats $(n=6)$ injected by saline from day 1 to day 14 and those $(n=6)$ injected by saline from day 2 to day 14 after CCI were both randomly selected for MWM trail. In sham group, 6 rats after sham operation were randomly selected for MWM trail. The apparatus consisted of a white interior circular pool $(0.5 \mathrm{~m}$ in depth and $1.0 \mathrm{~m}$ in diameter), which contained a submerged platform, that was divided into four virtual quadrants, with the addition of nontoxic white paint to make the water opaque. Water temperature was maintained at $25.0 \pm 0.5^{\circ} \mathrm{CC}$. All rats were accustomed to the water and MWM apparatus 1 day prior to initiation of navigational testing. In the hidden platform test, a submerged platform was positioned stochastically in the four quadrants, and its position was changed every day over the four daily trials using the same pattern on a given day for all experimental rats. Serial testing (four trials/day) was performed from day 17 [post injury day (PID) 17] to day 21 (PID21) after CCI injury. The maximum time rats were allowed to reach the submerged platform was $90 \mathrm{sec}$. If the rat could not find the platform within this time, it was placed on the platform by the experimenter and remained there for $10 \mathrm{sec}$.

In the visible platform test, two sets of trials were performed (from PID17 to PID18), with the platform raised $0.5 \mathrm{~cm}$ above the water and clearly marked. With regard to the spatial probe trial, on PID21, the platform was removed and the rats were placed in the same start location as in the acquisition trails ( $\sim 2 \mathrm{~h}$ after the fifth hidden platform trial) to swim freely for a maximum time of $60 \mathrm{sec}$ to reach the target quadrant zone, whereby the strength of the spatial memory retention would be assessed. All behavioral parameters of rats were tracked, recorded and analyzed using a MT-200 water maze video tracking system (Techman Software Co., Ltd., Chengdu, China).

Statistical analysis. Data were expressed as the mean \pm standard error of the mean. MWM and plasma cytokine data were analyzed using repeated measures analysis of variance (ANOVA) with Tukey's post hoc tests. With regard to the differences of mRNA levels between each group, statistical significance was accepted when the ratio of $2^{-\Delta \Delta \mathrm{Cq}}$ exceeded 1.7. One-way ANOVA with Tukey's post hoc tests was used for other data to evaluate the differences between certain groups. SPSS v. 18.0 statistical software (SPSS, Inc., Chicago, IL, USA) was used for statistical analyses. $\mathrm{P}<0.05$ was considered to indicate a statistically significant difference.

\section{Results}

$B B B$ permeability following CCI injury. To assess the effects of TMP on improving the breakdown of BBB after TBI, the extravasation of Evans Blue dye in the ipsilateral hemisphere was determined $24 \mathrm{~h}$ after CCI injury ( $\mathrm{n}=6$ for each group). Results demonstrated that, at $24 \mathrm{~h}$ after CCI injury, the Evans Blue dye extravasation in the ipsilateral cortex in the TMP and vehicle groups was significantly greater compared with the sham group $(\mathrm{P}<0.01$; Fig. 1$)$. In addition, compared with that of the vehicle group, the Evans Blue dye extravasation in

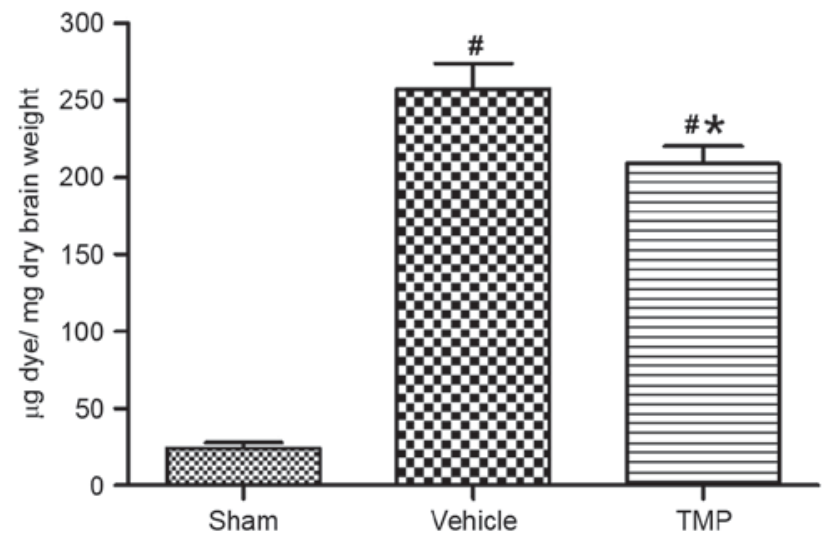

Figure 1. Assessment of BBB permeability following CCI injury. At $24 \mathrm{~h}$ after CCI injury, Evans Blue dye extravasation ( $\mu \mathrm{g}$ Evans Blue dye/mg dry weight of brain tissue) in the ipsilateral cortex in the TMP and vehicle groups were significantly greater than that of sham group. In addition, compared with that of the vehicle group, the Evans Blue dye extravasation in the ipsilateral cortex was significantly decreased in the TMP group. Data are presented as the mean \pm standard error of the mean. ${ }^{*} \mathrm{P}<0.05$ vs. the vehicle group; ${ }^{~} \mathrm{P}<0.01$ vs. the sham group. $\mathrm{BBB}$, blood-brain barrier; $\mathrm{CCI}$, controlled cortical impact; TMP, tetramethylpyrazine.

the ipsilateral cortex was significantly decreased in the TMP group $(\mathrm{P}<0.05$; Fig. 1).

Plasma values of IL-1 $\beta$ and TNF- $\alpha$ following CCI injury. In order to evaluate the potential effects of TMP on the circulating inflammatory responses following TBI, the plasma levels of IL-1 $\beta$ and TNF- $\alpha$ were detected within $24 \mathrm{~h}$ after CCI injury. IL-1 $\beta$ and TNF- $\alpha$ were both generally considered to be the markers of peripheral inflammatory cytokines. The ELISA assay ( $n=6$ for each group) demonstrated that, at 4, 12 and $24 \mathrm{~h}$ after CCI injury, respectively, the plasma levels of IL-1 $\beta$ and TNF- $\alpha$ in the TMP and vehicle groups were significantly higher compared with the sham group $(\mathrm{P}<0.05)$. Additionally, compared with that of vehicle group, the plasma levels of IL-1 $\beta$ and TNF- $\alpha$ at 4, 12 and $24 \mathrm{~h}$ after CCI injury were significantly decreased in the TMP group $(\mathrm{P}<0.05$; Fig. 2$)$.

mRNA and protein expression levels of $I L-1 \beta$ and TNF- $\alpha$ in the spleen following CCI injury. To evaluate the possible effects of TMP on the splenic expression of IL-1 $\beta$ and TNF- $\alpha$ following TBI, mRNA and protein expression levels of IL-1 $\beta$ and TNF- $\alpha$ in the spleen after CCI injury were assessed. RT-qPCR and western blotting demonstrated that, at $24 \mathrm{~h}$ after CCI injury, splenic mRNA and protein expression levels of IL-1 $\beta$ and TNF- $\alpha$ in the TMP and vehicle groups were significantly higher compared with the sham group $(\mathrm{P}<0.05$; Fig. 3). In addition, compared with that of the vehicle group, the splenic mRNA and protein expression levels of IL-1 $\beta$ and TNF- $\alpha 24 \mathrm{~h}$ after CCI injury were significantly decreased in the TMP group $(\mathrm{P}<0.05$; Fig. 3$)$.

Splenic nAChRa7-associated $N F-\kappa B$ and STAT3 signaling following CCI injury. To determine the potential effects of TMP on the splenic anti-inflammatory roles mediated by nAChRa7 and its downstream NF- $\mathrm{B}$ and STAT3 signaling, mRNA and protein expression levels of $n A C h R a 7, N F-\kappa B$ and STAT3 in the spleen following CCI injury were evaluated. 

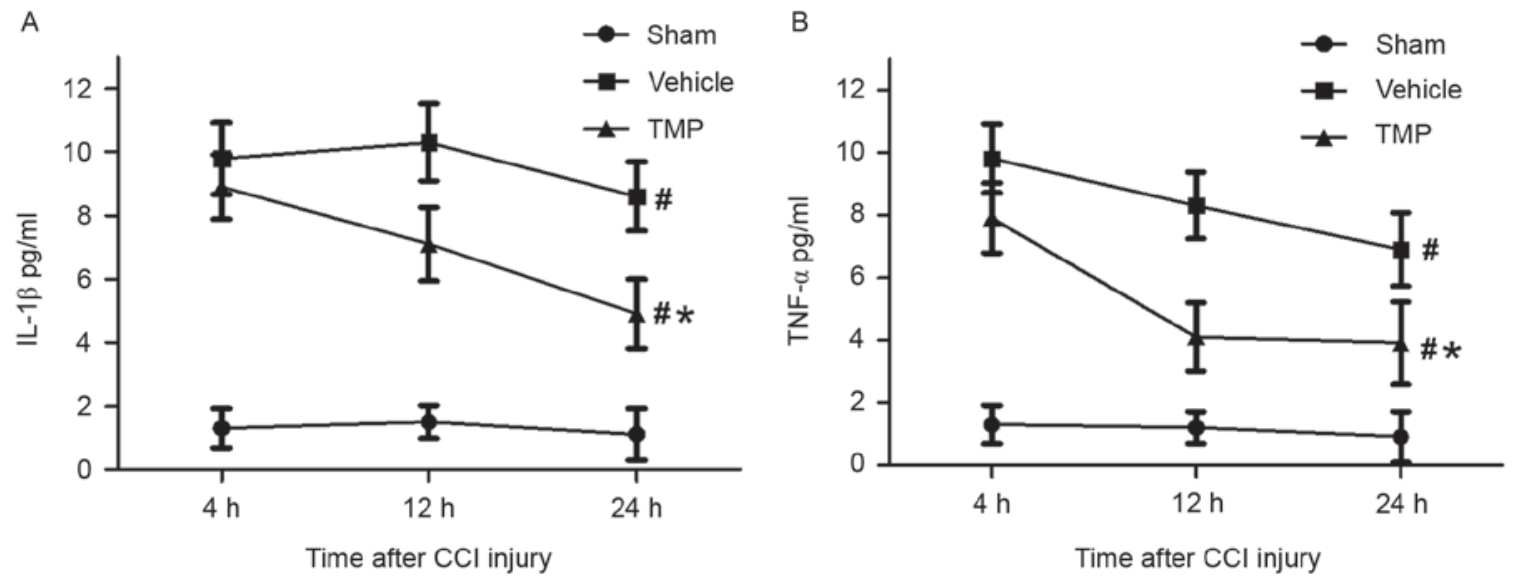

Figure 2. Plasma levels of IL-1 $\beta$ and TNF- $\alpha$ within $24 \mathrm{~h}$ after CCI injury. At 4, 12 and $24 \mathrm{~h}$ after CCI injury, respectively, the plasma levels of (A) IL-1 $\beta$ and (B) TNF- $\alpha$ in the TMP and vehicle groups were significantly higher than that of the sham group. Additionally, compared with the vehicle group, the plasma levels of IL-1 $\beta$ and TNF- $\alpha$ at 4, 12 and $24 \mathrm{~h}$ after CCI injury were significantly decreased in the TMP group. Data are presented as the mean \pm standard error of the mean. " $\mathrm{P}<0.05$ vs. the sham group at 4,12 and $24 \mathrm{~h}$; ${ }^{*} \mathrm{P}<0.05$ vs. the vehicle group at 4,12 and $24 \mathrm{~h}$. IL, interleukin; TNF, tumor necrosis factor; CCI, controlled cortical impact; TMP, tetramethylpyrazine.

A

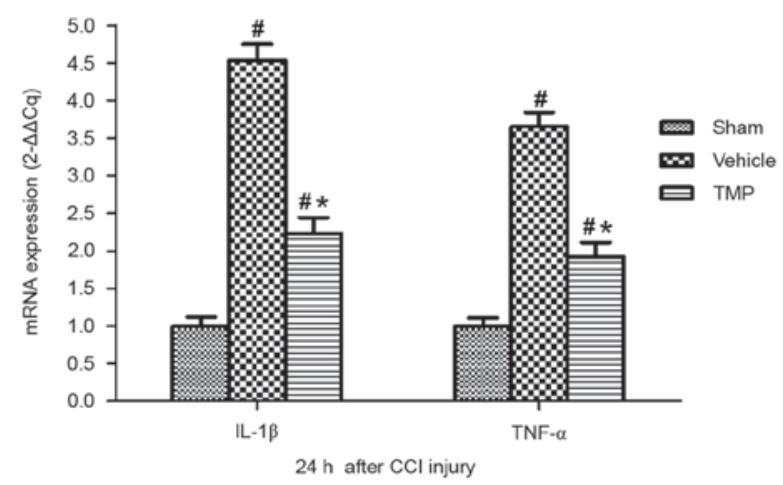

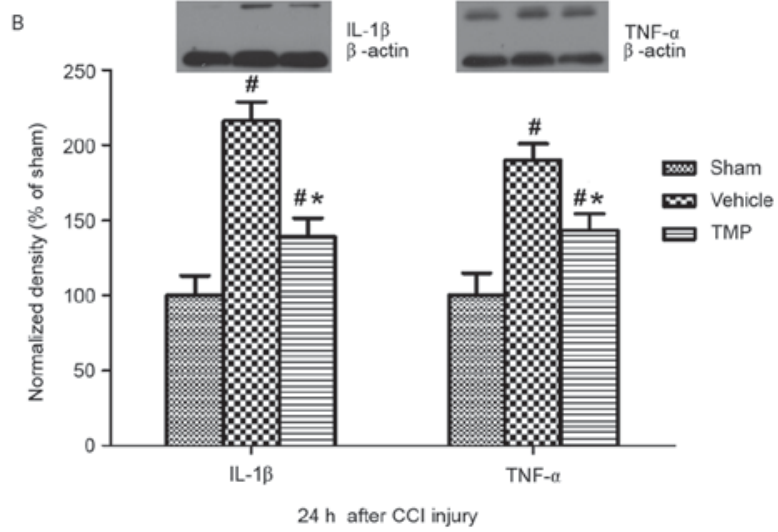

Figure 3. Splenic mRNA and protein expression levels of IL-1 $\beta$ and TNF- $\alpha 24 \mathrm{~h}$ after CCI injury. $24 \mathrm{~h}$ after CCI injury, the (A) mRNA and (B) protein expression levels of IL-1 $\beta$ and TNF- $\alpha$ in the spleen in the TMP and vehicle groups were significantly higher than that of the sham group. Additionally, compared to the vehicle group, the mRNA and protein expression levels of IL-1 $\beta$ and TNF- $\alpha$ in the spleen $24 \mathrm{~h}$ after CCI injury were significantly decreased in the TMP group. Data are presented as the mean \pm standard error of the mean. ${ }^{\prime} \mathrm{P}<0.05$ vs. the sham group; ${ }^{*} \mathrm{P}<0.05$ vs. the vehicle group. IL, interleukin; TNF, tumor necrosis factor; CCI, controlled cortical impact; TMP, tetramethylpyrazine.

RT-qPCR and western blotting demonstrated that, $24 \mathrm{~h}$ after CCI injury, the splenic mRNA and protein expression levels of

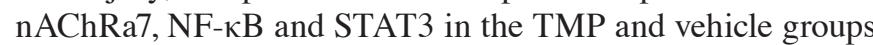
were significantly higher than that of the sham group $(\mathrm{P}<0.05$; Fig. 4). However, there were no significant differences in the expression levels of nAChRa7, NF- $\mathrm{nB}$ and STAT3 at $24 \mathrm{~h}$ after CCI injury between the TMP and vehicle groups. Furthermore, western blotting demonstrated that, $24 \mathrm{~h}$ after CCI injury, the splenic protein expression levels of $\mathrm{p}-\mathrm{NF}-\kappa \mathrm{B}$ and $\mathrm{p}-\mathrm{STAT} 3$ in the TMP and vehicle groups were significantly higher than that of the sham group $(\mathrm{P}<0.05$; Fig. 4). In addition, compared to that of the vehicle group, the splenic protein expression levels of p-NF- $\kappa$ B and p-STAT3 $24 \mathrm{~h}$ after CCI injury were significantly increased in the TMP group ( $\mathrm{P}<0.05$; Fig. 4).

Spatial learning and memory following CCI injury. To determine the beneficial effects of TMP on cognitive recovery after TBI, spatial learning and memory following CCI injury were assessed. MWM results demonstrated that, in the visible platform test, at PID17 and PID18 after CCI injury, there were no significant differences in latency time between the TMP, vehicle and sham groups (Fig. 5). In the hidden platform test, from PID17-21 after CCI injury, the latency times in the TMP and vehicle groups were significantly increased compared with those of the sham group $(\mathrm{P}<0.05$; Fig. 5). Additionally, compared with those of the vehicle group, the latency times in the hidden platform test were significantly decreased in the TMP group $(\mathrm{P}<0.05)$. When the injection administration on the same day as CCI procedure was not applied in the TMP and vehicle groups [TMP (2-14) and vehicle (2-14)], a significant difference in latency time in the hidden platform test was not detected. Furthermore, in the spatial probe trial, on PID21 after CCI injury ( $2 \mathrm{~h}$ after the fifth hidden platform trial), the TMP and vehicle groups demonstrated significantly worse performances than the sham group ( $\mathrm{P}<0.05$; Fig. 5). Additionally, compared to that of the vehicle group, the TMP group displayed significantly improved performance $(\mathrm{P}<0.05)$. When the injection administration on the same day of CCI 
A

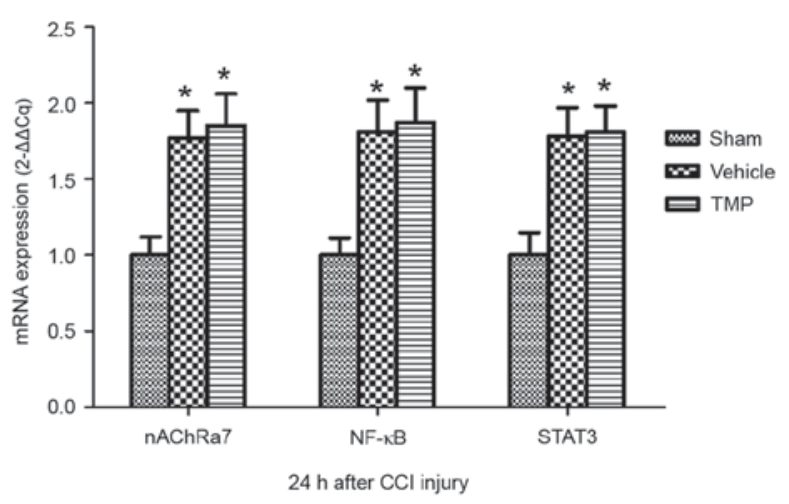

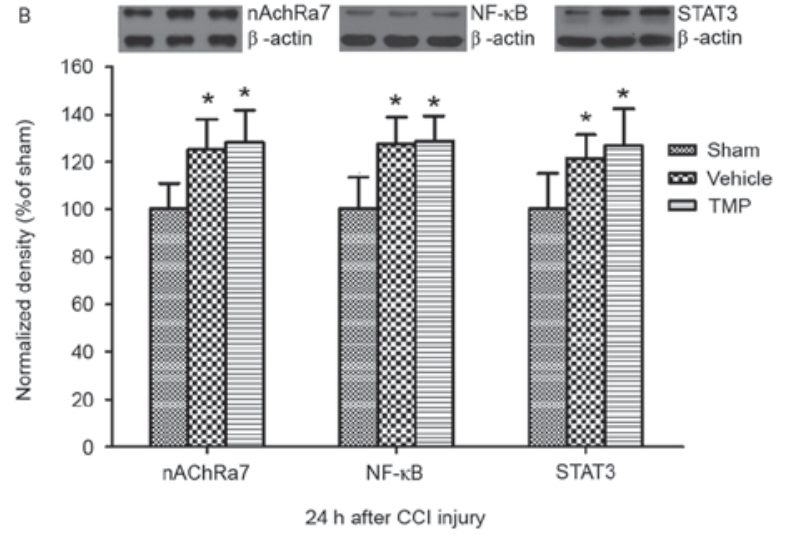

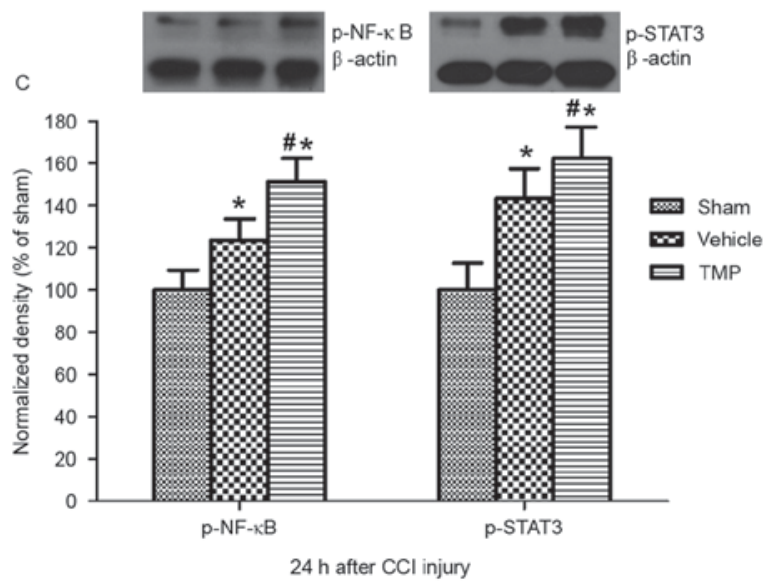

Figure 4. Splenic mRNA and protein expression levels of nAChRa7, NF- $\mathrm{kB}$ and STAT3, and protein expressions levels of p-NF-kB and p-STAT3, $24 \mathrm{~h}$ after CCI injury. At $24 \mathrm{~h}$ after CCI injury, the (A) mRNA and (B) protein expression levels of nAChRa7, NF-кB and STAT3 in the spleen in the TMP and vehicle groups were significantly higher than that of the sham group. However, there were no significant differences in these levels between the TMP and vehicle groups. At $24 \mathrm{~h}$ after CCI injury, the splenic protein expression levels of (C) p-NF-kB and p-STAT3 in the TMP and vehicle groups were significantly higher than that of the sham group. In addition, compared to the vehicle group, the splenic protein expression levels of p-NF- $\mathrm{kB}$ and p-STAT3 were significantly increased in the TMP group. Data are presented as the mean + standard error of the mean. ${ }^{*} \mathrm{P}<0.05$ vs. the sham group; ${ }^{*} \mathrm{P}<0.05$ vs. the vehicle group. nAChRa7, nicotinic acetylcholine receptor $\alpha 7$; NF, nuclear factor; STAT3, signal transducer and activator of transcription 3; p, phosphorylated; CCI, controlled cortical impact; TMP, tetramethylpyrazine.

procedure was not applied in the TMP and vehicle group, a significant difference in performance in the spatial probe trial was not detected (Fig. 5).

\section{Discussion}

While the explicit mechanistic underpinnings remain largely unclear, the complex processes under which secondary injury cascades are induced by initial primary mechanical insults are now extensively considered to be the central dogma in the pathogenesis of TBI (27). Previous research has revealed that, in the early phase of secondary injuries, the regional cerebral blood flow, as well as tissue oxygenation, in the ipsilateral hemisphere following TBI is prominently decreased $(28,29)$. As such, we proposed that the pathological mechanisms underlying TBI and ischemic brain injury should overlap, at least in part, during the early events. In fact, cumulating evidence has demonstrated that TMP could exert explicit therapeutic effects on improving the clinical outcomes of ischemic brain injury, such as ischemic stroke (30). Accordingly, the present study hypothesized that TMP may also provide some benefits for treating TBI. The present study demonstrated that the application of TMP following TBI was able to: i) Effectively reduce $\mathrm{BBB}$ permeability; ii) actively inhibit peripheral inflammation associated with stimulation of splenic cholinergic activity; and iii) potentially improve the cognitive recovery concerning spatial learning and memory.

The abnormal dysfunction of BBB after TBI has been widely considered to be a transient process, which could critically deteriorate various other post-injury processes so as to cause poor life-long outcomes (15). Therefore, to effectively improve and correct $\mathrm{BBB}$ damage is paramount for treating TBI. The results of the present study demonstrated that the application of TMP could reduce BBB permeability after $24 \mathrm{~h}$ of CCI injury. Notably, following TBI, circulating pro-inflammatory cytokines, such as IL- $1 \beta$ and TNF- $\alpha$, dramatically increased within hours (18). Furthermore, it was reported that intraperitoneal injection of pro-inflammatory cytokines to experimental animals could worsen the pathological consequences of TBI (31). In the present study, plasma levels of IL-1 $\beta$ and TNF- $\alpha$ within 24 h of CCI injury were significantly elevated, and these levels were significantly decreased by TMP. Thus, TMP may essentially reduce the permeability of BBB after TBI, which may be closely related to the inhibition 


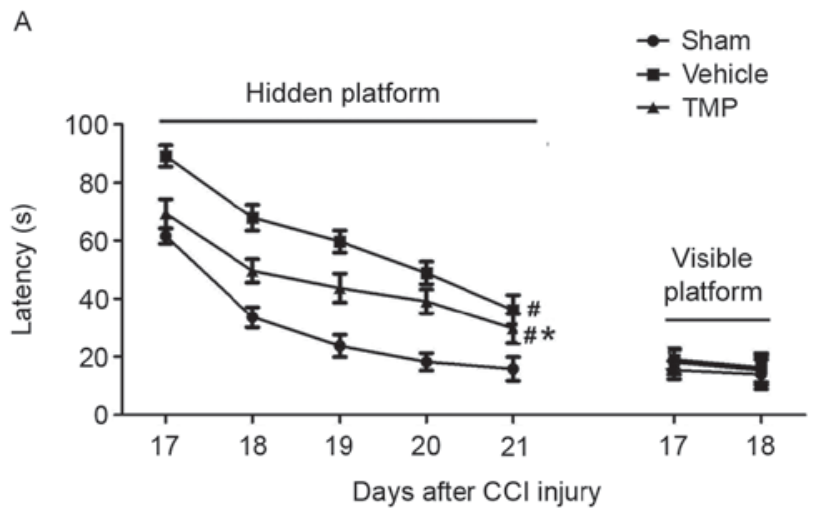

B
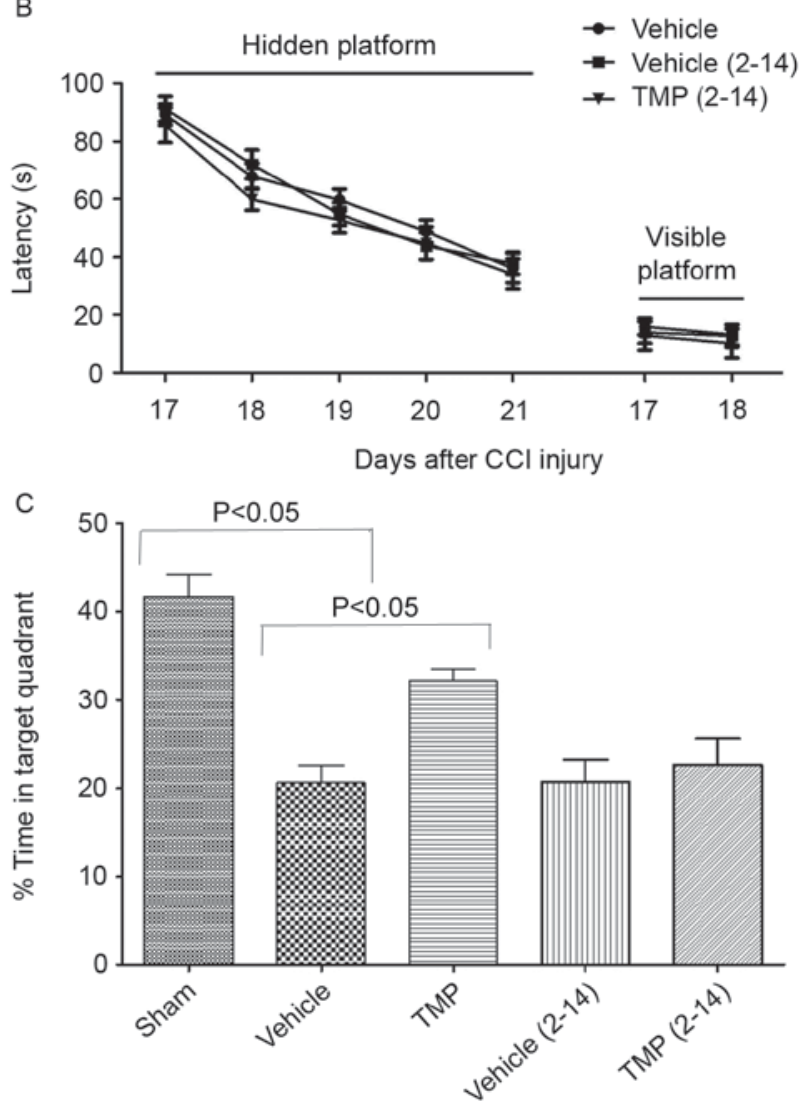

Figure 5. Spatial learning and memory after CCI injury. (A) In the visible platform test, there were no significant differences in latency time between the TMP, vehicle and sham groups. In the hidden platform test, from PID17-21 after CCI injury, the latency time in the TMP and vehicle groups were significantly increased compared with the sham group. Additionally, compared with the vehicle group, the latency times were significantly decreased in the TMP group. (B) When the first two injection administrations were deprived in the TMP and vehicle groups [TMP (2-14) and vehicle (2-14)], there were no significant differences in latency time between the TMP (2-14), vehicle and vehicle (2-14) groups. Data are presented as the mean \pm standard error of the mean. (C) In the spatial probe trial, the TMP and vehicle groups displayed significantly worse performance than the sham group. Additionally, compared with the vehicle group, the TMP group displayed significantly improved performance. When the first two injection administrations were not applied in the TMP and vehicle groups, there were no significant differences in performance between the TMP (2-14), vehicle and vehicle (2-14) groups. Data are presented as the mean + standard error of the mean. ${ }^{~} \mathrm{P}<0.05$ vs. the sham group at PID17-21; ${ }^{*} \mathrm{P}<0.05$ vs. the vehicle group at PID17-21. CCI controlled cortical impact; TMP, tetramethylpyrazine; PID, post injury day.

of the systemic inflammatory status. In fact, the BBB is composed of cerebrovascular endothelium, astrocytic endfeet and extracellular matrix (14). Accruing evidence has indicated that TBI would exert various detrimental insults on the basement membrane, endothelial cells and inter-endothelial tight junctions by certain proteases, so as to increase BBB permeability (15). Recent findings have indicated that TMP could increase the expression of tight junction proteins, such as occludin and claudin-5, in ischemic brain injury (17). However, whether the beneficial effects of TMP on reducing BBB permeability after TBI should be associated with the aforementioned pathways is unknown and requires further investigation.

Although researchers have studied the neuroinflammation triggered by TBI and its detrimental effects on BBB permeability, one study has ascertained the defined contribution of systemic inflammatory responses in the pathogenesis of BBB breakdown (21). Novel findings have demonstrated that vagus nerve activity, particularly the splenic efferent nerve, could actively reduce systemic inflammatory responses (32). Furthermore, it was demonstrated that splenectomy could markedly reduce BBB permeability triggered by TBI (22). Thus, the vagus innervated spleen may serve as a peripheral anti-inflammatory feedback mechanism to the TBI-triggered neuroinflammation in order to decrease the breakdown of BBB. In the present study, it was demonstrated that protein and mRNA expression levels of IL-1 $\beta$ and TNF- $\alpha$ in the spleen were significantly decreased by TMP after CCI injury. As such, we proposed that the decreased plasma levels of IL-1 $\beta$ and TNF- $\alpha$ were, at least in part, due to the inhibitory effects on their expression in the spleen by TMP in TBI.

Notably, novel findings have implicated that the aforementioned splenic anti-inflammatory effects were mediated by the activation of $\mathrm{nAChRa7}$ on splenic macrophages so as to decrease the circulating levels of pro-inflammatory cytokines (23). The present results indicated that protein and mRNA expression levels of splenic nAChRa7 were significantly elevated following CCI injury. However, the TBI-triggered nAChRa7 expression could not be further increased by the application of TMP. Subsequently, by assessing the downstream signaling of nAChRa7, it was demonstrated that, compared to that of vehicle group, the expression levels of $\mathrm{p}-\mathrm{NF}-\kappa \mathrm{B}$ and $\mathrm{p}-\mathrm{STAT} 3$ in the spleen were significantly increased by TMP after CCI injury. Meanwhile, although expression levels of splenic $\mathrm{NF}-\kappa \mathrm{B}$ and STAT3 were significantly elevated in CCI injury, the TBI-triggered expressions of $\mathrm{NF}-\kappa \mathrm{B}$ and STAT3 could not be further increased by the application of TMP. This indicated that both NF- $\kappa \mathrm{B}$ and STAT3 signaling were further activated by TMP after CCI injury (Fig. 4). Other research has suggested that nAChRa7-mediated neuroprotection is initiated by the activation of Janus kinase 2, followed by the stimulation of protein kinase B, NF- $\mathrm{B}$ and STAT3 (33). Thus, it should be indicated that, after TBI, TMP could further enhance the downstream signaling of nAChRa7, which may be closely associated with the inhibited expression of IL-1 $\beta$ and TNF- $\alpha$ in the spleen. Therefore, it was implicated that TMP could stimulate the nAChRa7-mediated cholinergic activity of the splenic macrophages, whereby the protein and mRNA expression levels of IL- $1 \beta$ and TNF- $\alpha$ would be inhibited, so as to elicit the peripheral anti-inflammatory effects on reducing BBB permeability triggered by TBI.

Using MWM tests to detect the spatial learning and memory has been conducted for nearly 30 years, and is widely 
accepted in the research of behavioral neuroscience for laboratory rodents (34). In order to remove the interference by visual and/or motor changes after TBI, we first assessed the visible platform test. Results demonstrated that there were no significant differences of the latency time between each group. Following this, the hidden platform test demonstrated that applying TMP after CCI injury could significantly shorten the latency time. Additionally, spatial probe trials indicated that using TMP after CCI injury could significantly improve the performance to reach the target quadrant. These data implicated that the impaired spatial acquisition and memory retention triggered by TBI could be, at least in part, improved by TMP. Furthermore, it was demonstrated that if the initial treatment of TMP within $24 \mathrm{~h}$ after CCI injury was not received, the latency time of spatial acquisition and performance of memory retention would not improve compared with the vehicle group. Thus, we proposed that the prompt application of TMP in the early 'time window' for treating TBI is essential for the recovery of cognitive functions, such as spatial learning and memory, which may be closely related to the reduction of $\mathrm{BBB}$ permeability. As the mechanistic underpinnings are extremely complex in the regulation of spatial learning and memory, it is unclear which and how the abnormal structures and/or altered cellular and molecular pathways triggered by TBI would be changed by TMP. Thus, this requires further investigation.

Here, we have to notice that, in the viewpoint of chemistry, TMP is structurally similar to that of nicotine. In fact, previous research has indicated that TMP could effectively inhibit the activity of acetylcholinesterase (35). However, it is unclear whether TMP could increase the local acetylcholine by its inhibitory effects on acetylcholinesterase activity and further activate nAChRa7 on splenic macrophages. Additionally, one important feature of $\mathrm{nAChRa7}$ is that the receptor would be rapidly desensitized after its activation (36). However, whether the desensitization characteristics of $\mathrm{nAChRa7}$ could be regulated by TMP was not assessed in this study. Furthermore, activation of nAChRa7 could instantly inhibit the secretion of IL-1 $\beta$ and TNF- $\alpha$, mediated by intracellular calcium-triggered signaling (37). Nonetheless, whether, and to what extent, TMP may regulate the intracellular calcium homeostasis in splenic macrophages triggered by TBI is unknown. Undoubtedly, the aforementioned pathways regulated by TMP require further study in future research.

In conclusion, the present study first verified the beneficial effects of TMP on reducing BBB permeability triggered by TBI, which was closely associated with the enhancement of splenic cholinergic anti-inflammatory effects. Accordingly, the significance of this contribution would be two-fold: Firstly, regarding its low price and clinical safety, TMP may serve as a potential candidate for clinical therapy for TBI; and secondly, to effectively promote the peripheral cholinergic anti-inflammatory effects in the early 'time window' may be critically important for functional recovery in treating TBI.

\section{References}

1. Cecil S, Chen PM, Callaway SE, Rowland SM, Adler DE and Chen JW: Traumatic brain injury: Advanced multimodal neuromonitoring from theory to clinical practice. Crit Care Nurse 31: 25-36; quiz 37, 2011.
2. Hyder AA, Wunderlich CA, Puvanachandra P, Gururaj G and Kobusingye OC: The impact of traumatic brain injuries: A global perspective. Neuro Rehabilitation 22: 341-353, 2007.

3. Centers for Disease Control and Prevention (CDC): CDC grand rounds: Reducing severe traumatic brain injury in the United States. MMWR Morb Mortal Wkly Rep 62: 549-552, 2013.

4. Langlois JA, Rutland-Brown W and Wald MM: The epidemiology and impact of traumatic brain injury: A brief overview. J Head Trauma Rehabil 21: 375-378, 2006.

5. Bhatnagar S, Iaccarino MA and Zafonte R: Pharmacotherapy in rehabilitation of post-acute traumatic brain injury. Brain Res 1640: 164-179, 2016.

6. Giacino JT, Whyte J, Bagiella E, Kalmar K, Childs N, Khademi A, Eifert B, Long D, Katz DI, Cho S, et al: Placebo-controlled trial of amantadine for severe traumatic brain injury. N Engl J Med 366: 819-826, 2012.

7. Ran X, Ma L, Peng C, Zhang H and Qin LP: Ligusticum chuanXiong Hort: A review of chemistry and pharmacology. Pharm Biol 49: 1180-1189, 2011.

8. Li W, Tang Y, Chen Y and Duan JA: Advances in the chemical analysis and biological activities of chuanxiong. Molecules 17: 10614-10651, 2012.

9. Shang YH, Tian JF, Hou M and Xu XY: Progress on the protective effect of compounds from natural medicines on cerebral ischemia. Chin J Nat Med 11: 588-595, 2013.

10. Jia J, Zhang X, Hu YS, Wu Y, Wang QZ, Li NN, Wu CQ, Yu HX and Guo QC: Protective effect of tetraethyl pyrazine against focal cerebral ischemia/reperfusion injury in rats: Therapeutic time window and its mechanism. Thromb Res 123: 727-730, 2009.

11. Kao TK, Chang CY, Ou YC, Chen WY, Kuan YH, Pan HC, Liao SL, Li GZ and Chen CJ: Tetramethylpyrazine reduces cellular inflammatory response following permanent focal cerebral ischemia in rats. Exp Neurol 247: 188-201, 2013.

12. Sun Y, Yu P, Zhang G, Wang L, Zhong H, Zhai Z, Wang L and Wang Y: Therapeutic effects of tetramethylpyrazine nitrone in rat ischemic stroke models. J Neurosci Res 90: 1662-1669, 2012.

13. Zhang C, Teng F, Tu J and Zhang D: Ultrasound-enhanced protective effect of tetramethylpyrazine against cerebral ischemia/reperfusion injury. PLoS One 9: e113673, 2014.

14. Zhao Z, Nelson AR, Betsholtz C and Zlokovic BV: Establishment and dysfunction of the blood-brain barrier. Cell 163: 1064-1078, 2015.

15. Alluri H, Wiggins-Dohlvik K, Davis ML, Huang JH and Tharakan B: Blood-brain barrier dysfunction following traumatic brain injury. Metab Brain Dis 30: 1093-1104, 2015.

16. Algattas $\mathrm{H}$ and Huang JH: Traumatic brain injury pathophysiology and treatments: Early, intermediate, and late phases post-injury. Int J Mol Sci 15: 309-341, 2013.

17. Tan F, Fu W, Cheng N, Meng DI and Gu Y: Ligustrazine reduces blood-brain barrier permeability in a rat model of focal cerebral ischemia and reperfusion. Exp Ther Med 9: 1757-1762, 2015.

18. Das M, Mohapatra S and Mohapatra SS: New perspectives on central and peripheral immune responses to acute traumatic brain injury. J Neuroinflammation 9: 236, 2012.

19. Turtzo LC, Lescher J, Janes L, Dean DD, Budde MD and Frank JA: Macrophagic and microglial responses after focal traumatic brain injury in the female rat. J Neuroinflammation 11: $82,2014$.

20. Rosas-Ballina M and Tracey KJ: The neurology of the immune system: Neural reflexes regulate immunity. Neuron 64: 28-32, 2009.

21. Walker PA, Shah SK, Jimenez F, Gerber MH, Xue H, Cutrone R, Hamilton JA, Mays RW, Deans R, Pati S, et al: Intravenous multipotent adult progenitor cell therapy for traumatic brain injury: Preserving the blood brain barrier via an interaction with splenocytes. Exp Neurol 225: 341-352, 2010.

22. Li M, Li F, Luo C, Shan Y, Zhang L, Qian Z, Zhu G, Lin J and Feng H: Immediate splenectomy decreases mortality and improves cognitive function of rats after severe traumatic brain injury. J Trauma 71: 141-147, 2011.

23. Dash PK, Zhao J, Kobori N, Redell JB, Hylin MJ, Hood KN and Moore AN: Activation of alpha 7 cholinergic nicotinic receptors reduce blood-brain barrier permeability following experimental traumatic brain injury. J Neurosci 36: 2809-2818, 2016.

24. Chang CY, Kao TK, Chen WY, Ou YC, Li JR, Liao SL, Raung SL and Chen CJ: Tetramethylpyrazine inhibits neutrophil activation following permanent cerebral ischemia in rats. Biochem Biophys Res Commun 463: 421-427, 2015. 
25. Kim M, Kim SO, Lee M, Lee JH, Jung WS, Moon SK, Kim YS, Cho KH, Ko CN and Lee EH: Tetramethylpyrazine, a natural alkaloid, attenuates pro-inflammatory mediators induced by amyloid $\beta$ and interferon- $\gamma$ in rat brain microglia. Eur J Pharmacol 740: 504-511, 2014.

26. Livak KJ and Schmittgen TD: Analysis of relative gene expression data using real-time quantitative PCR and the 2(-Delta Delta C(T)) Method. Methods 25: 402-408, 2001.

27. Mustafa AG and Alshboul OA: Pathophysiology of traumatic brain injury. Neurosciences (Riyadh) 18: 222-234, 2013.

28. Kenney K, Amyot F, Haber M, Pronger A, Bogoslovsky T, Moore $\mathrm{C}$ and Diaz-Arrastia R: Cerebral vascular injury in traumatic brain injury. Exp Neurol 275: 353-366, 2016

29. Baumann G, Travieso L, Liebl DJ and Theus MH: Pronounced hypoxia in the subventricular zone following traumatic brain injury and the neural stem/progenitor cell response. Exp Biol Med (Maywood) 238: 830-841, 2013.

30. Lin JB, Zheng CJ, Zhang X, Chen J, Liao WJ and Wan Q: Effects of tetramethylpyrazine on functional recovery and neuronal dendritic plasticity after experimental stroke. Evid Based Complement Alternat Med 2015: 394926, 2015.

31. Utagawa A, Truettner JS, Dietrich WD and Bramlett HM: Systemic inflammation exacerbates behavioral and histopathological consequences of isolated traumatic brain injury in rats. Exp Neurol 211: 283-291, 2008.
32. Olofsson PS, Rosas-Ballina M, Levine YA and Tracey KJ: Rethinking inflammation: Neural circuits in the regulation of immunity. Immunol Rev 248: 188-204, 2012.

33. Marrero MB and Bencherif $\mathrm{M}$ : Convergence of alpha 7 nicotinic acetylcholine receptor-activated pathways for anti-apoptosis and anti-inflammation: Central role for JAK2 activation of STAT3 and NF-kappaB. Brain Res 1256: 1-7, 2009.

34. Brown MA and Sharp PE: Simulation of spatial learning in the Morris water maze by a neural network model of the hippocampal formation and nucleus accumbens. Hippocampus 5: 171-188, 1995.

35. Zhang C, Wang SZ, Zuo PP, Cui X and Cai J: Protective effect of tetramethylpyrazine on learning and memory function in D-galactose-lesioned mice. Chin Med Sci J 19: 180-184, 2004.

36. Palma E, Conti L, Roseti C and Limatola C: Novel approaches to study the involvement of $\alpha 7-n A C h R$ in human diseases. Curr Drug Targets 13: 579-586, 2012.

37. Westerlund A, Björklund U, Rönnbäck L and Hansson E: Long-term nicotine treatment suppresses IL-1 $\beta$ release and attenuates substance P- and 5-HT-evoked $\mathrm{Ca}^{2+}$ responses in astrocytes. Neurosci Lett 553: 191-195, 2013. 